\title{
Individual differences in metabolism predict coping styles in fish
}

\author{
Catarina I.M. Martins ${ }^{\mathrm{a}, *}$, Maria F. Castanheira ${ }^{\mathrm{a}}$, Sofia Engrola ${ }^{\mathrm{a}}$, \\ Benjamín Costas ${ }^{\mathrm{a}, \mathrm{b}}$, Luís E.C. Conceição ${ }^{\mathrm{a}}$ \\ a Centro de Ciências do Mar (CCMar), Universidade do Algarve, Campus de Gambelas, 8005-139 Faro, Portugal \\ b Centro Interdisciplinar de Investigação Marinha e Ambiental (CIIMAR), Universidade do Porto, 4050-123 Porto, Portugal
}

\section{A R T I C L E I N F O}

\section{Article history:}

Accepted 13 December 2010

Available online 15 January 2011

\section{Keywords:}

Personality

Respirometry

Resting metabolic rate

Cortisol

Behaviour

Individual variation

\begin{abstract}
A B S T R A C T
Studies on metabolism usually rely on measurements of oxygen consumption obtained in respirometry chambers. Despite rigorous standardization there is still considerable interindividual variation in metabolic rates which is often ignored. Furthermore, housing in respirometry chambers implies handling and exposure to a new environment, conditions known to be a source of stress in animals. The extent of stress response is known to be dependent on an individual's coping style. Therefore, the extent of individual variation in respirometry data may be related to the expression of coping styles. The goal of this study is to determine whether the variation in oxygen consumption after a stressor is the resultant of an individual's coping style. Our approach was to determine oxygen consumption in fish immediately after transfer to respiratory chambers and during the subsequent $22 \mathrm{~h}$. In addition, the same individuals were subjected to 3 respirometry runs ( $22 \mathrm{~h}$ each) to address the question whether habituation occurs when fish are used in multiple runs and whether habituation potential is linked to coping styles. The same individuals were then subjected to an acute stressor and the escape behaviour and plasma cortisol were determined to assess coping style. Taken as a whole, the findings of this study suggest that individual variation in oxygen consumption in respirometry chambers are related to the expression of coping styles: individuals with higher oxygen consumption at the start and throughout the measurement period were shown to react passively (longer latency to escape) in a confinement stress. In addition, successive respirometry runs should not be assumed to result in habituation, as depending on an individual's coping style either habituation (in the case of passive copers) or sensitization (in the case of active copers) can occur.
\end{abstract}

(c) 2010 Elsevier B.V. All rights reserved.

\section{Introduction}

In animals, including fish, individual variation in phenotypic traits is no longer considered as simply the result of statistical noise but rather a reflection of important biological processes. Coping styles (or personalities, temperament, behaviour syndromes, bold/shy continuum) are one of such processes and are defined as a coherent set

\footnotetext{
* Corresponding author. Tel.: +351 289800 900x7167; fax: +351289800 069 .

E-mail address: cimartins@ualg.pt (C.I.M. Martins).
}

of behavioural and physiological stress responses which is consistent over time and which is characteristic to a certain group of individuals (Koolhaas et al., 1999). Over the past years, several studies have shown that individual variation in a range of behaviour and physiological traits in fish exposed to stress reflect divergent coping styles (Øverli et al., 2005, 2006, 2007; Frost et al., 2007; Kristiansen and Ferno, 2007; Silva et al., 2010). These studies have gained increasing attention as coping styles have been shown to be key players in fish welfare (Huntingford and Adams, 2005; Huntingford et al., 1999), health and diseases susceptibility (Fevolden et al., 1992; MacKenzie et al., 2009), performance traits (Martins, 2005) and more recently for the 
interpretation of molecular data (MacKenzie et al., 2009). The approaches to study coping styles in fish have differed between using strains of fish selected for cortisol responsiveness (e.g. Øverli et al., 2002) or using the whole range of variation within a population (e.g. Silva et al., 2010). In either case, the results suggest that active copers, as opposed to passive copers can be behaviourally characterised by (1) actively trying to escape from a stressor (Silva et al., 2010), (2) showing higher feed motivation after transfer to a new environment (Øverli et al., 2007), (3) being more risk taking and exploratory when exposed to novelty (Øverli et al., 2006; Frost et al., 2007; MacKenzie et al., 2009) and (4) being more aggressive (dominant) during aggressive encounters (Øverli et al., 2004). In addition, active copers exhibit typical neuroendocrine responses such as (1) lower hypothalamus-pituitary-interrenal (HPI) activity (Silva et al., 2010), (2) lower HPI reactivity (Øverli et al., 2005), (3) higher sympathethic reactivity and lower parasympathetic reactivity (measured as opercular beat rate, e.g. Verbeek et al., 2008), and (4) higher testosterone activity (Koolhaas et al., 1999), as compared to passive copers.

Behavioural and physiological responses underlying coping with stress are energy demanding (Barton and Schreck, 1987) and have been suggested to be part of an individual's maintenance requirement (Martins et al., 2005, 2006, 2008). In vertebrates, energy metabolism is often measured as resting metabolic rate (i.e. lowest metabolic level of a fasted animal) in respirometry chambers (CruzNeto and Bozinovic, 2004). These chambers represent a novel environment to which individuals are not adapted. In addition to the new environmental conditions (e.g. area, volume, colour of the chamber, light, surrounding sounds) also the social environment is different as in most cases fish originating from a group are housed alone in the chamber to allow individual measurements. In order to minimize variation in the data set researchers try to perform measurements in controllable conditions, using individuals with similar body weights, ignore the data collected during the first hours of housing and perform multiple runs to allow habituation (i.e. reduced response to repeated stimulation not attributable to fatigue or sensory adaptation (Domjan, 2003), to the chamber (Careau et al., 2008). However, despite rigorous standardization and calibration of the respiratory systems equipment there is still considerable inter-individual variation in resting metabolic rate which is often ignored. Mean values are used as the only relevant information while the variation around the mean is often ignored and assumed to be a consequence of measurement errors. This approach, however, has been questioned by a number of authors such as Careau et al. (2008) who underlies the lack of scientific effort to address individual variation in resting metabolic rates by posing the question "how can we explain the fact that a difference as small as $10 \%$ (often less!) between the mean resting metabolic rate of two populations, groups or treatments can be subjected of pages of discussion while variation as large as $200 \%$ in resting metabolic rate among individuals does not merit mention?"

In fish, several studies have been done addressing differences in metabolic rates between different populations and how these differences relate to coping with environmental conditions (e.g. Seppänen et al., 2008; Grabowski et al., 2009). However, to the best of our knowledge no study has ever addressed how far individual differences in resting metabolic rate reflect intra-specific coping styles. Housing in respirometry chambers implies handling and exposure to a new environment, both conditions known to be a source of stress in a variety of fish species (Martins et al., 2006; Höglund et al., 2007; Ramsay et al., 2009; Silva et al., 2010). As a consequence some individuals may exhibit a fight or flight reaction while others a freezelike reaction. These responses are expected to have an energetic cost. As suggested by Careau et al. (2008) animal personality creates a spectrum of metabolic rates that underpins the energy spent on both behaviour and neuroendocrine responses associated with coping. In fish, very little is known concerning the link between metabolism and coping styles. Martins et al. (2005, 2006, 2008) using residual feed intake as a proxy of maintenance requirement showed a link between feeding motivation, aggressiveness and cortisol response after acute stress (variables reflecting coping styles in African catfish) and metabolism. However, these studies did not measure metabolism directly using respiratory chambers. Furthermore, the general assumption that habituation occurs after the same individuals is exposed to successive respiratory runs still needs to be validated in fish. In addition, whether different coping styles differ in habituation potential was never investigated in fish. Ellenberg et al. (2009) showed, in birds, that passive copers showed a higher habituation potential as compared to active (more aggressive) animals.

The goal of this study is to determine whether the individual variation in oxygen consumption after a stressor is the resultant of an individual's coping style. Our approach was to determine oxygen consumption in fish immediately after transfer to the respiratory chambers and during the subsequent $22 \mathrm{~h}$. In addition, the same individuals were subjected to 3 respirometry runs to address the question whether habituation occurs when fish are used in multiple runs and whether habituation potential is linked to coping styles. The same individuals were then subjected to an acute stressor and the plasma cortisol and escape behaviour determined to assess coping style.

Senegalese sole Solea senegalensis was used as our experimental model. This species is known to exhibit coping styles (Silva et al., 2010) and to stand motionless for long periods of time minimizing the contribution of swimming activity to the measured oxygen consumption values.

\section{Methods}

\subsection{Fish, feeding and housing conditions}

Twelve Senegalese sole (S. senegalensis) juveniles with an average start body weight of $18.5 \pm 2.9 \mathrm{~g}$ were used. Fish were obtained from natural spawning of wild broodstock and reared according to standard larval and juvenile rearing protocols (Dinis et al., 1999). Fish were kept at the Ramalhete facility at the CCMAR/University of Algarve (Faro, Portugal) in two $12.6 \mathrm{~L}$ flat-bottomed fibreglass tanks (70 cm length $\times 30 \mathrm{~cm}$ width $\times 6 \mathrm{~cm}$ depth) in a semi-closed 
seawater recirculation system equipped with a biofilter and UV filter, at a constant flow rate in the tanks of $109 \mathrm{~L} / \mathrm{h}$ (eight renovations per hour). The water renewal in the recirculation system was $19.5 \%$ per day. Water quality was maintained within the optimal range for the species and was monitored daily.

A 12L:12D photoperiod was maintained with day break set at $0600 \mathrm{~h}$; light intensity was kept at 12 lux. Fish were fed ad libitum, once a day (at 0930) with commercial diet (Aquagold $2 \mathrm{~mm}$, Sorgal SA, Ovar, Portugal; 44\% crude protein, $14 \%$ crude fat, $8 \%$ ash, $2.5 \%$ crude fibres, $1.0 \%$ phosphorus).

Fish were individually tagged with colour codes. Before tagging, fish were randomly transferred to a $10 \mathrm{~L}$ bucket, filled with $5 \mathrm{~L}$ of seawater and anaesthetic, 2phenoxyethanol (Sigma-Aldrich, Germany; 1:150, v/v). Three different colours of water based paint (Acualux TITAN "al agua" colores satinados, Industrias TITAN, S.A., Spain) were used for colour marks. The colours were injected in the sole blind side in the opposite area to the abdomen and parallel to muscle direction. The paint was injected using sterile syringes (ONCE $1 \mathrm{ml}$ ) and non-pyrogenic needles (Terumo Neolus $25 \mathrm{G} \times 5 / 8^{\prime \prime}$; $0.5 \mathrm{~mm} \times 16 \mathrm{~mm}$, Terumo Europe N.V., Belgium).

\subsection{Experimental procedures}

\subsubsection{Respirometry}

Oxygen consumption was determined by flow through respirometry on days 85, 106 and 113 after fish arrival to the Ramalhete facilities. These measurements were done to determine whether habituation (decrease in oxygen consumption between runs) occurs in all individuals. Before transfer to the respiratory chambers fish were left unfed for $24 \mathrm{~h}$. Measurements started immediately after transfer and continued for $22 \mathrm{~h}$. The body weight of each individual was determined immediately after the metabolic measurements.

The flow-through respirometry system consisted in individual metabolic chambers $(2.3 \mathrm{~L} ; 19.0 \mathrm{~cm}$ diameter and $8 \mathrm{~cm}$ depth). Each fish was placed individually in a chamber. Each chamber had a water inlet and a water outlet. Water inlet was always at oxygen saturation level and oxygen concentration at outlet was measured by a poligraphic microelectrode (model 8-730, Microelectrodes Inc., USA) connected to a PC using the Oxilogger 2009 software (João Reis, CCMAR, Faro, Portugal). The saturation level of oxygen was maintained in the water reservoir and a peristaltic pump (ISMATEC, model ISM920A, Switzerland), controlled the water flow of each chamber. At the beginning of each cycle the oxygen dissolved in water was measured during a 30 s period in order to calibrate the software. This calibration was followed by a 120 s washing step of seawater from the next chamber before the start of the next measurement period ( $30 \mathrm{~s}$ ). This step was always done before and after the measurement of each chamber. The data was collected through a dynamic mean of 6 measurements ( $5 \mathrm{~s}$ each) during a total period of $30 \mathrm{~s}$. The water temperature was always measured in the outlet water of each chamber by a temperature probe.

The following parameters were determined using the oxygen consumption data (in $\mu \mathrm{mol} \mathrm{O}_{2} / \mathrm{g}$ body weight/h) (based on Careau et al. (2008): (1) oxygen consumption during the first $10 \mathrm{~min}$ (from now on first $10 \mathrm{~min} M R$ ), corresponding to the average oxygen consumption per individual during the first $10 \mathrm{~min}$ immediately after the fish entered the respiratory chamber, (2) maximum oxygen consumption ( $\max M R$ ), corresponding to the highest value of oxygen consumption, per individual, during the $22 \mathrm{~h}$ measurement period, (3) lowest oxygen consumption ( $\min M R)$, corresponding to the lowest value of oxygen consumption, per individual, during the $22 \mathrm{~h}$ measurement period, (4) the difference between the max and min oxygen consumption (span MR), (5) the average of all measurements of oxygen consumption (average $M R$ ), per individual, during the $22 \mathrm{~h}$ measurement period, (6) the average of the period of the lowest oxygen consumption measurement (resting metabolic rate, $R M R$ ) and (7) the coefficient of variation of all measurements of oxygen consumption ( $C V M R$ ), per individual, during the $22 \mathrm{~h}$ measurement period.

The lowest oxygen consumption in run 1 took place between 1530 and $1800 \mathrm{~h}$ (3.5-6h after transfer to respirometry chambers, Fig. 1). Oxygen consumption

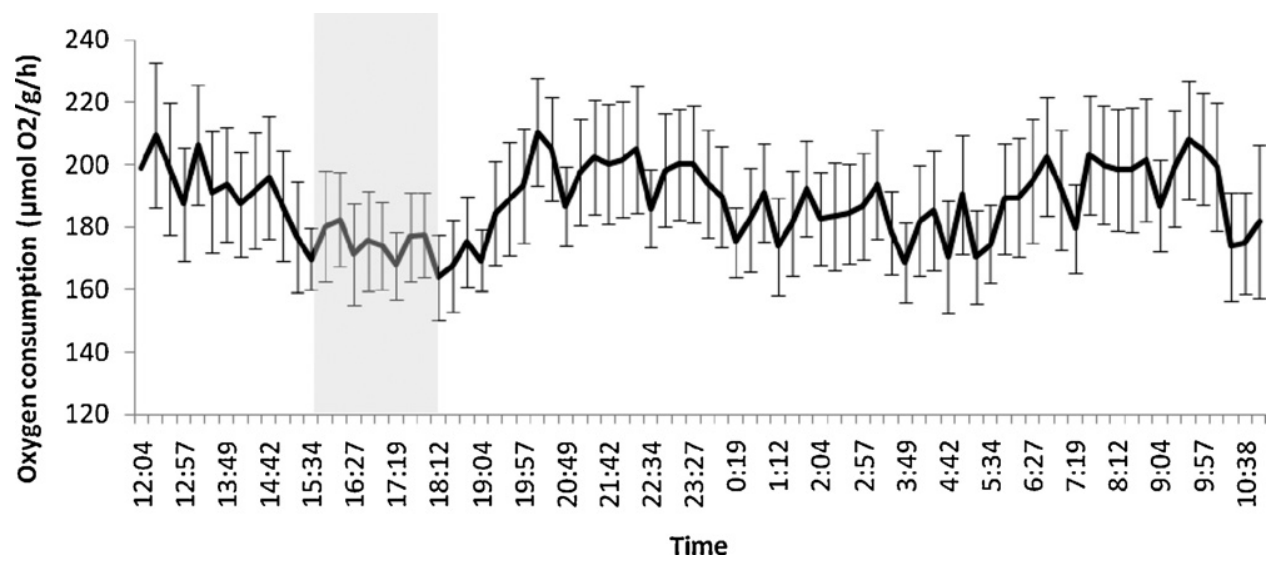

Fig. 1. Variation in oxygen consumption over a period of $22 \mathrm{~h}$ in 12 juveniles of Solea senegalensis. Shadow area indicates the period of lowest oxygen consumption that was used to obtain resting metabolic rate. 

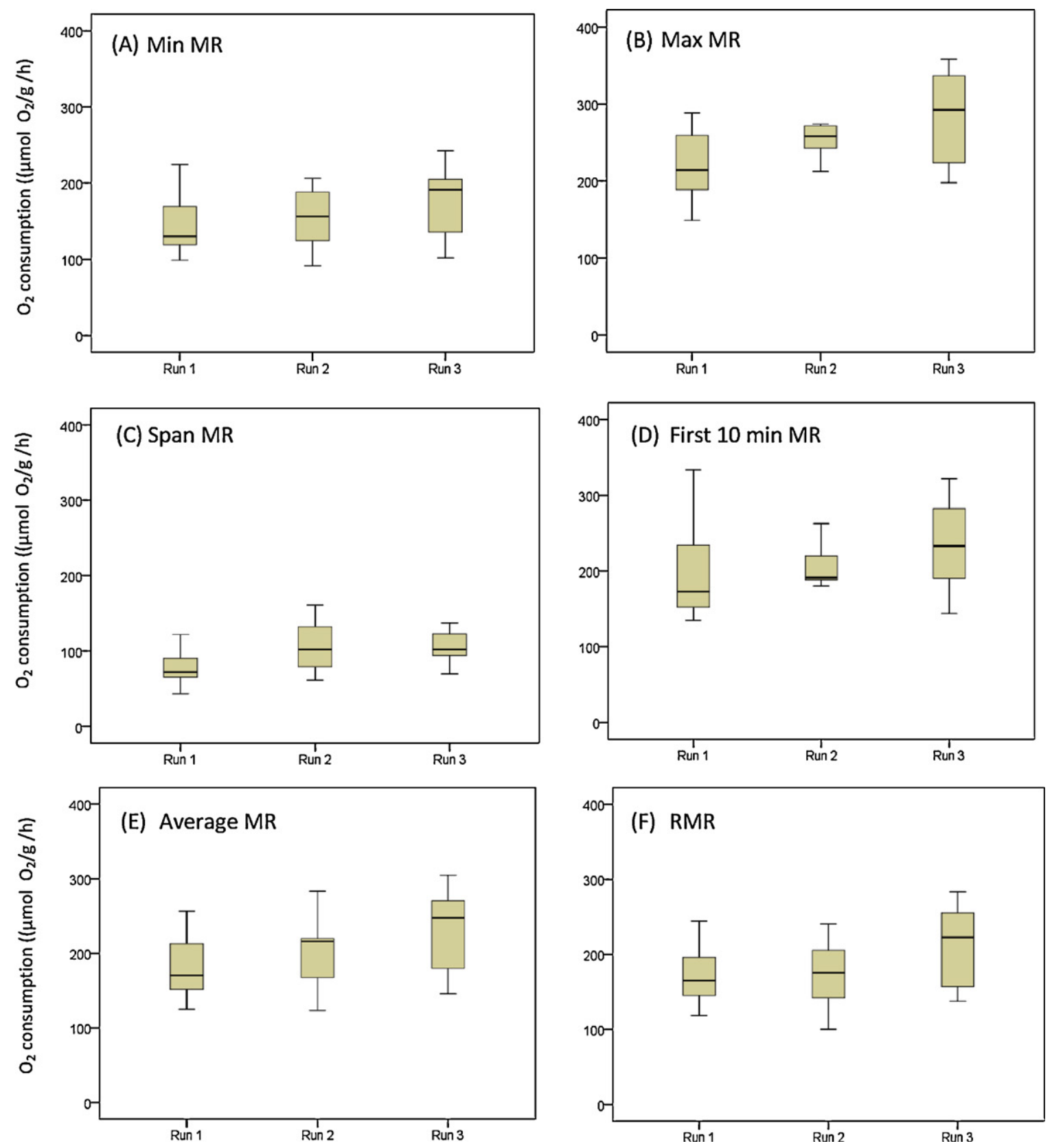

Fig. 2. Box plots of $\min M R(A)$, max MR (B), span MR, (C), first 10 min MR (D), average MR (E) and RMR (F) for the 3 respirometry runs ( $n=12$ in each run). The box includes observations from the 25 th to the 75 th percentile, the horizontal line within the box represents the median value. Lines outside the box represent the 10 th and the 90 th percentiles.

obtained during this period was used as indicative of resting metabolic rate (in $\mu \mathrm{mol} \mathrm{O}_{2} / \mathrm{g}$ body weight $/ \mathrm{h}$ ).

\subsubsection{Coping styles}

After the 3 respiratory runs, fish were kept under standardized conditions until day 163 when an acute stress was induced by holding each fish individually in a net outside the water for $3 \mathrm{~min}$ (Silva et al., 2010). Plasma samples were collected $1 \mathrm{~h}$ after the start of the stress test for latter determination of cortisol via radioimmunoassay. While in the net, fish behaviour was also assessed by direct observation. Two parameters were determined: (1) the total number of escape attempts, i.e. number of body moments that resulted in an elevation of the body from the net and (2) the latency for the first escape attempt, i.e. the time (in s) taken by each fish to show an escape attempt. One individual did not show escape behaviour resulting in $n=11$ for the behavioural data.

Blood sampling was performed in anaesthetized fish (2-phenoxyethanol, 1:500, v/v). Blood was withdrawn 
from the caudal vein using heparinised syringes $(1 \mathrm{ml})$ and non-pyrogenic needles (Terumo Neolus $25 \mathrm{G} \times 5 / 8^{\prime \prime}$; $0.5 \mathrm{~mm} \times 16 \mathrm{~mm})$. A heparin solution $(1000 \mathrm{U} / \mathrm{ml}$; grade I-A: from porcine intestinal mucosa; Sigma-Aldrich, Germany) was prepared in $\mathrm{NaCl} 0.9 \%$ and used as anticoagulant in the syringes and needles. The blood collection lasted less than $3 \mathrm{~min}$ to avoid a cortisol increase due to handling. After sampling, blood was centrifuged in a HERMLE centrifuge (Z233 M-2) with HERMLE rotor (220.87 VO 5/6) at $1500 \mathrm{~g}$ during $8 \mathrm{~min}$ at room temperature. Plasma was removed with micropipettes and stored in micro-tubes at $-80^{\circ} \mathrm{C}$ until further analysis. In six samples, the resulting plasma from centrifugation was coagulated and was not considered for analysis, resulting in $n=6$ for plasma samples.

\subsection{Cortisol analysis}

Plasma cortisol levels were measured with a commercially available competitive binding Coat-A-Count ${ }^{\circledR}$ Cortisol kit (SIEMENS Medical Solutions Diagnostics, Los Angeles, CA, USA) as described by Irwin et al. (1999). Briefly, $50 \mu \mathrm{l}$ of each sample to be assayed was transferred into an Ab-Coated tube and $1 \mathrm{ml}$ of ${ }^{125}$ I Cortisol added. The tubes were then incubated for $45 \mathrm{~min}$ at $37^{\circ} \mathrm{C}$ in a water bath. The contents of all tubes were decanted, and allowed to drain for $5 \mathrm{~min}$ before being read on a gamma counter (2470 WIZARD $^{2 \mathrm{TM}}$, PerkinElmer ${ }^{\mathrm{TM}}$, Inc., Zaventem, Belgium) for $1 \mathrm{~min}$. A calibration curve was constructed on logit-log graph paper and used to convert results from percent binding cortisol to concentration $\left(\mathrm{ng} \mathrm{ml}^{-1}\right)$. The Coat-A-Count cortisol antiserum cross-reacts $100 \%$ with cortisol, $11.4 \%$ with 11 -deoxycortisol, $0.98 \%$ with cortisone, $0.94 \%$ with corticosterone and $0.02 \%$ with progesterone.

\subsection{Statistics}

Statistical analyses were performed using SPSS 16.0 for windows. Relationships between variables were investigated using Pearson correlation after the normality of data was verified (number of escape attempts and cortisol were log transformed). A new variable was created, delta first $10 \mathrm{~min} M R$, corresponding to the changes (increase or decrease) in oxygen consumption between successive runs. Possible differences between $\max M R$, min $M R$, span $M R$, first 10 min $M R$, average $M R$ and $R M R$ over the successive respirometry runs were tested using repeated measures design. Mauchly's test was used to assess the assumption of sphericity and the Bonferroni test for making pairwise comparisons (based on Field, 2000). The comparison Statistical significance was taken at $P<0.05$.

\section{Results}

\subsection{Individual variation in oxygen consumption}

Fig. 2 depicts the pronounced individual variation in different oxygen consumption parameters obtained after transfer to respirometry chambers. Despite there is an overall increase of the median for all the oxygen consumption parameters considered, there was no statistical
A
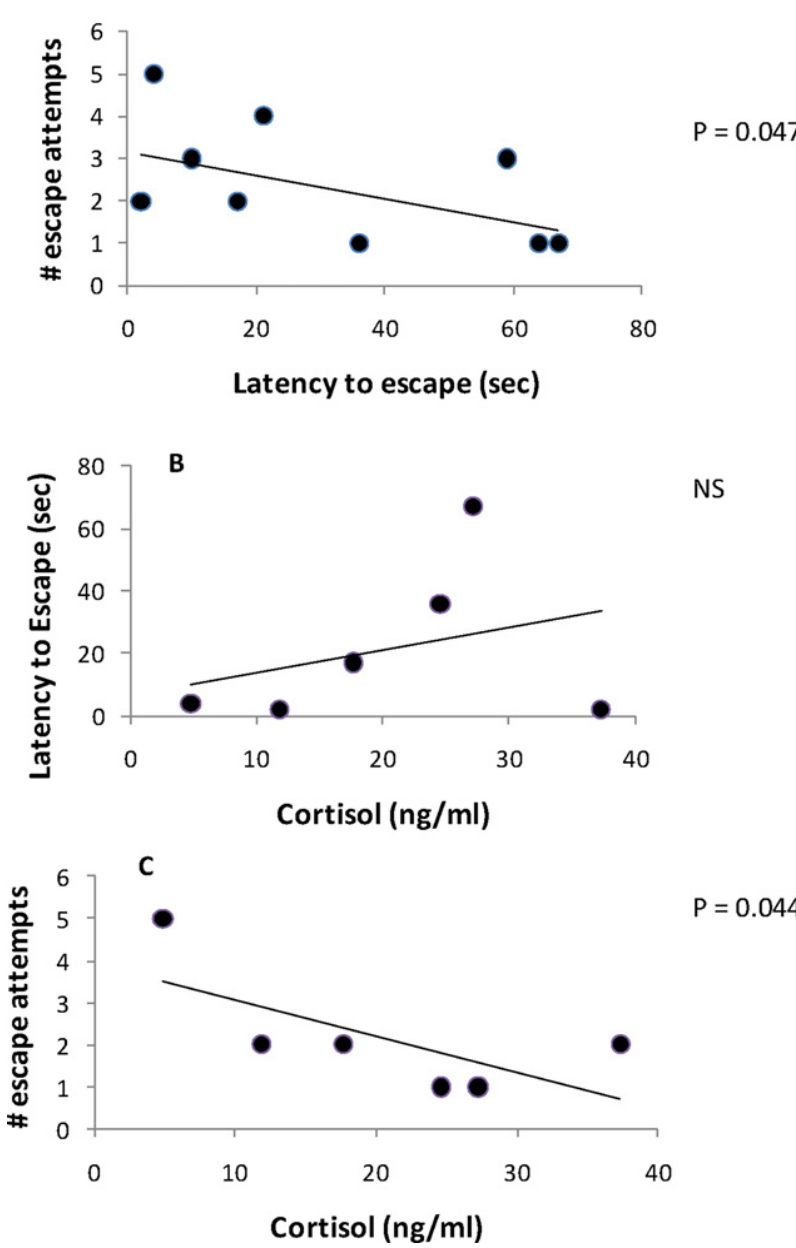

Fig. 3. Relationship between plasma cortisol and escape behaviours (latency and number) when juveniles of Senegalse sole are exposed to an acute confinement stress (note that in (A), 2 data set ( $x, y: 67.1$ and 2.2) are repeated twice, resulting in a visual observation of 9 points; total $n$ is 11).

difference between the mean of $\min M R, \max M R$, first $10 \mathrm{~min} M R$, average $M R$ and $R M R$ between successive respirometry runs. Only span $M R$ showed a significant difference $\left(F_{2,22}=6.024, P=0.008\right)$ with run 3 showing a significant higher value than run $1(P=0.009)$.

\subsection{Stress test: linking cortisol and escape behaviour}

The two behavioural parameters measured during the stress test were significantly correlated $(P=0.047$, $r_{p}=-0.61$, Fig. 3): individuals taking longer to escape (higher latency to escape) were also the ones that exhibited the lower number of escape attempts, suggesting a passive response towards the acute confinement stress. The cortisol levels obtained after the stress test were negatively correlated with the number of escape attempts $(P=0.044$, $r_{p}=-0.82$ ) indicating that high cortisol responders try to escape less often than low cortisol responders. 


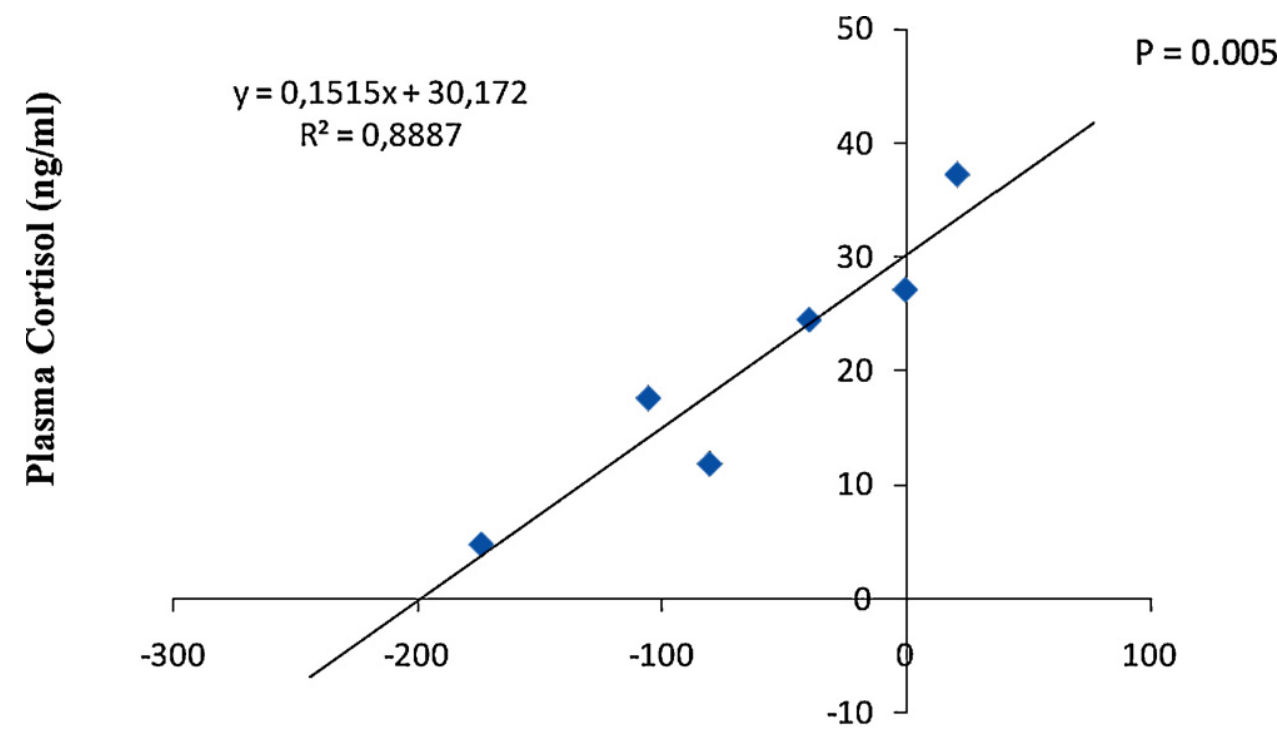

\section{Delta first $10 \min M R\left(\mu \mathrm{mol} \mathrm{O}_{2} / \mathrm{g} / \mathrm{h}\right)$}

Fig. 4. Relationship between delta first $10 \mathrm{~min} M R\left(\mathrm{O}_{2}\right.$ consumption in the first 10 min of run $1-\mathrm{O}_{2}$ consumption in the first 10 min of run 3$)$ and plasma cortisol obtained after an acute confinement stress test.

\subsection{Multiple runs in respirometry trials: habituation or increased stress?}

Fig. 4 depicts the relationship between the variable delta first 10 min MR (between runs 1 and 3) and the behaviour and cortisol response during and after the stress test, respectively. Some individuals show positive deltas while others negative deltas suggesting that in some individuals the oxygen consumption decreases between runs while in other it increases, respectively.

There was no significant correlation between the behaviour and cortisol parameters and the change in oxygen consumption between runs 1 and 2 or between runs 2 and 3. However, there was a significant correlation between change in oxygen consumption between trials 1 and 3 and the cortisol response. This shows that individuals that increase their oxygen consumption between respiratory runs (resulting in negative deltas) exhibit a low cortisol response when subjected to an acute confinement stress.

\subsection{Is individual variation in oxygen consumption linked} with coping styles?

Only the oxygen consumption measurements obtained in the first respirometry run were used to correlate with the behaviour and stress response. This decision was based on the fact that successive runs have different consequences in terms of oxygen consumption for different individual (some habituate while others do not - see Section 3.3).

Table 1 summarizes the relationships between the metabolic variables and the behavioural and cortisol response obtained after the acute stress. Cortisol was not significantly correlated with any of the metabolic variables. However, latency to escape was significantly correlated with first $10 \mathrm{~min} M R\left(P=0.032 ; r_{p}=0.706\right), \max M R$ $\left(P=0.032, r_{p}=0.644\right)$, average $M R\left(P=0.026, r_{p}=0.663\right)$ and RMR $\left(P=0.024, r_{p}=0.669\right)$, suggesting that the longer an individual takes to exhibit an escape attempt the higher the oxygen consumption is during the first $10 \mathrm{~min}$ in the respirometry chamber, when considering the oxygen consumption during the $22 \mathrm{~h}$ measurement period and when considering the period corresponding to the resting metabolic rate. The number of escape attempts was negatively correlated with the coefficient of variation of all oxygen consumption measurements, suggesting that individuals who tried to escape more often showed less variation during the measurement period.

\section{Discussion}

This study shows that when individuals are transferred to respirometry chambers they exhibit a pronounced

Table 1

Correlation between plasma cortisol and escape behaviour after an acute confinement stress and metabolic rate parameters (all expressed in $\mu \mathrm{mol} \mathrm{O}_{2} / \mathrm{g} / \mathrm{h}$ ). NS means not significant.

\begin{tabular}{|c|c|c|c|c|c|c|}
\hline & First 10 min MR & Max MR & Span MR & Average MR & RMR & CV MR \\
\hline Cortisol (ng ml-1) & NS & NS & NS & NS & NS & NS \\
\hline Latency to escape (sec) & $P=0.015$ & $P=0.032$ & NS & $P=0.026$ & $P=0.024$ & $P=0.051$ \\
\hline & $r_{p}=0.706$ & $r_{p}=0.644$ & & $r_{p}=0.663$ & $r_{p}=0.669$ & $r_{p}=0.600$ \\
\hline \# Escape attempts & NS & NS & NS & NS & NS & $\begin{array}{l}P=0.043 \\
r_{p}=-0.618\end{array}$ \\
\hline
\end{tabular}


individual variation in a variety of oxygen consumption measurements that are often ignored in metabolic studies. Furthermore, the same individuals also exhibit a pronounced individual variation in behaviour and cortisol response when subjected to an acute confinement stress used to predict coping styles in fish (Øverli et al., 2007; Silva et al., 2010). Despite the limited sample size for cortisol data, the values obtained as well as the relationship with the escape behaviour during the confinement stress is in agreement with a previous study done in Senegalese sole (Silva et al., 2010). In the present study, we showed for the first time that the variation in oxygen consumption after transfer to respirometry chambers and behavioural/cortisol response after confinement stress are related, and that the variation observed in oxygen consumption can be suggested as a predictor of coping styles in fish. Individuals that consume less oxygen after transfer into a respirometry chamber and during the $22 \mathrm{~h}$ measurement period were also the individuals that reacted sooner to a confinement stress (typical from active copers).

But how can oxygen consumption in respiratory chamber predict divergent coping strategies? Careau et al.(2008) suggested that transfer to respirometry chamber can be seen as transfer to a novel environment and that differences in oxygen consumption may reflect different exploratoryactivity levels. In this case active copers are expected to start exploring the novel environment faster but for shorter periods as compared to passive (or reactive) copers. Such behavioural response of fast-explorers would be reflected in higher MR at the start of a run and lower RMR while slowexplores would be expected to sustain a moderate MR and less variable MR throughout the run (Careau et al., 2008). In the present study, we found that individuals that react as active copers when subjected to a confinement stress (by showing lower latency to escape) are the individuals that show the lowest RMR. However, these individuals were also the ones showing the lowest oxygen consumption during the first $10 \mathrm{~min}$ after transfer to the respirometry chambers and the individuals with the lowest coefficient of variation in MR. Therefore, in our study, oxygen consumption in respirometry chambers seem not to be related to exploratory behaviour as this would have implied a higher oxygen consumption (probably linked with swimming activity) at the start of the run in active copers. Two possible explanations for the lack of increased MR in active copers after transfer to the respiratory chamber can be suggested. Firstly, exploratory behaviour in Senegalese sole may not be relevant as a personality trait (similar suggestion has been raised in other species e.g. Minderman et al., 2009). Senegalese sole can be considered as a calm species as opposed to other species that exhibit hyper-activity when transferred to respiratory chambers. Although we did not measure activity level in the respirometry chamber, we observed that all individuals remained quite in the bottom of the respirometry chambers. Secondly, transfer to respirometry chamber implies netting the fish out of their home tank (and social environment) into a new (and socially isolated) environment. Netting has been shown to induce a significant stress response in Senegalese sole (Silva et al., 2010) and different type of stressors have been shown to impair exploratory activity in non-fish species
(Garcia-Marquez and Armario, 1987; D’Aquila et al., 2000). Therefore, one may argue that the oxygen consumption measurements obtained immediately after transfer to the respirometry chamber may be an indicative more of a stress response rather than of exploratory behaviour. In this case, active copers by showing lower oxygen consumption during the first $10 \mathrm{~min}$ after transfer as well as during the $22 \mathrm{~h}$ measurement period suggest a quicker recovery from transfer than passive copers. This is supported by other studies that showed a quicker recovery of active copers (measured by increased appetite) after transfer into a new environment (Höglund et al., 2007; Øverli et al., 2007).

Respiratory responses have been used as a physiological measure of emotional arousal in humans (Boiten et al., 1994) and more recently in animals (Reefmann et al., 2009). Appraisal refers to how an individual evaluates/perceives/interprets a stimulus. When our fish were transferred to the respirometry chambers it is likely that the way the new environment was interpreted differed among individuals. Different factors can influence appraisal such as prior experiences, age, gender, and coping styles (Lazarus, 1991). From the results of this study we can suggest that passive copers exhibit a higher arousal when exposed to a new physical and social environment. Whether such arousal reflects an (negative) emotional valence needs to be further investigated. Although the link between coping styles, appraisal and emotions was never investigated in fish, studies on other species suggest that passive (or reactive) individuals are more prone to negative emotions such as fear and exhibit attention bias towards negative stimuli, as opposed to active individuals (Richards et al., 1992; Derryberry and Reed, 1994; Amin et al., 2004; Cockrem, 2007). Further research is needed to shed light on whether the same principles that link personality, appraisal and coping in higher vertebrates also apply in fish.

RMR is used in metabolism studies assuming that it represents the oxygen consumption necessary for minimal activity and maintenance requirements (Careau et al., 2008). RMR is obtained after a few hours individuals have been transfer to the metabolic chamber and it is often assumed that a stress response is no longer influencing the oxygen consumption measurements. The results from this study show that coping style is playing an important role in explaining differences in RMR and that the interpretation of results should take the individual's coping style in consideration. Recently, the incorporation of an individual's coping style for the interpretation of data that typically only considers average values revealed a significant influence of coping styles on the interpretation of data (MacKenzie et al., 2009).

The results of this study also show that the approach used in metabolic studies to subject the same individuals to successive respirometry runs to allow them to adapt is not valid for all individuals. Individuals that showed a higher cortisol response after a confinement stress (typical from passive individuals) were also the individuals that exhibited a decrease in oxygen consumption from runs 1 to 3 suggesting that habituation took place. However, for the majority of the individuals the oxygen consumption increased from runs 1 to 3 (suggesting an increased sensitization to the respirometry housing rather 
than habituation). The increase was higher in individuals with lower cortisol response after the confinement stress (typical from active individuals). Such result should be interpret with caution due to the limited sample size, however, this observation is in agreement with other studies in higher vertebrates showing that passive copers habituate faster than active copers (e.g. Ellenberg et al., 2009). Several authors have suggested that passive copers tend to be more observant and more prone to change on the basis of experience, whereas active copers are more likely to adopt routines (Koolhaas et al., 1999; Bolhuis et al., 2004; Groothuis and Carere, 2005; Quinn and Cresswell, 2005). These differences seem to be part of a higher behavioural plasticity of passive copers as compared to active copers. It should be noted, however, that this plasticity seems to be context dependent. Frost et al. (2007) for instance, showed that bold fish appear to be more flexible in changing their behaviour when observing other individuals with opposing personality traits, as compared to shy individuals.

In conclusion, this study shows that individuals of Senegalese sole exhibit pronounced individual variation in oxygen consumption while in respirometry chambers and that this variation seems to be predictive of coping styles. Furthermore, coping styles appear to influence whether an individual habituates or becomes more sensitized to successive respirometry runs and therefore should be taken into account in metabolic studies.

\section{Acknowledgements}

C.I.M. Martins, S. Engrola and B. Costas were supported by grants provided by the Foundation for Science and Technology, Portugal (SFRH/BPD/42015/2007, SFRH/BPD/42051/2008 and SFRH/BD/38697/2007, respectively). This study benefited from funding by the ECOAQUA5.E (programme POCTEP, co-funded by FEDER, European Commission). The authors would like to thanks to João Reis for hardware and software development and technical assistance during the respirometry runs.

\section{References}

Amin, Z., Constable, R.T., Canli, T., 2004. Attentional bias for valenced stimuli as a function of personality in the dot-probe task. J. Res. Pers. 38, 15-23.

Barton, B.A., Schreck, C.B., 1987. Influence of acclimation temperature on interrenal and carbohydrate stress responses in juvenile chinook salmon (Oncorhynchus tshawytscha). Aquaculture 62, 299310

Boiten, F.A., Frijda, N.H., Wientjes, C.J.E., 1994. Emotions and respiratory patterns: review and critical analysis. Int. J. Psychophysiol. 17, 103-128.

Bolhuis, J.E., Schouten, W.G.P., Leeuw, J.A.D., Schrama, J.W., Wiegant, V.M., 2004. Individual coping characteristics, rearing conditions and behavioural flexibility in pigs. Behav. Brain Res. 152, 351-360.

Careau, V., Thomas, D., Humphries, M.M., Reale, D., 2008. Energy metabolism and animal personality. Oikos 117, 641-653.

Cockrem, J.F., 2007. Stress, corticosterone responses and avian personalities. J. Ornithol. 148, S169-S178.

Cruz-Neto, A.P., Bozinovic, F., 2004. The relationships between diet quality and basal metabolic rate in endotherms: insights from intraspecific analysis. Physiol. Biochem. Zool. 77, 877-889.

D'Aquila, P.S., Peana, A.T., Carboni, V., Serra, G., 2000. Exploratory behaviour and grooming after repeated restraint and chronic mild stress: effect of desipramine. Eur. J. Pharmacol. 399, 43-47.
Derryberry, D., Reed, M.A., 1994. Temperament and attention: orienting toward and away from positive and negative signals. J. Pers. Soc. Psychol. 66, 1128-1139.

Dinis, M.T., Ribeiro, L., Soares, F., Sarasquete, C., 1999. A review on the cultivation potential of Solea senegalensis in Spain and in Portugal. Aquaculture 176, 27-38.

Domjan, M., 2003. The Principles of Learning and Behavior, 5th ed. Wadsworth/Thomson Learning, Belmont, California.

Ellenberg, U., Mattern, T., Seddon, P.J., 2009. Habituation potential of yellow-eyed penguins depends on sex, character and previous experience with humans. Anim. Behav. 77, 289-296.

Fevolden, S.E., Refstie, T., Røed, K.H., 1992. Disease resistance in rainbow trout (Oncorhynchus mykiss) selected for stress response. Aquaculture $104,19-29$.

Field, A., 2000. In: Breakwell, G., Leeuw, J., O’Muircheartaigh, C., Saris, W., Schuman, H., van Meter, K. (Eds.), Discovering Statistics using SPSS for Windows. Sage Publications, London.

Frost, A.J., Winrow-Giffen, A., Ashley, P.J., Sneddon, L.U., 2007. Plasticity in animal personality traits: does prior experience alter the degree of boldness? Proc. R. Soc. Biol. 274, 333-339.

Garcia-Marquez, C., Armario, A., 1987. Chronic stress depresses exploratory activity and behavioral performance in the forced swimming test without altering ACTH response to a novel acute stressor. Physiol. Behav. 40, 33-38.

Grabowski, T.B., Young, S.P., Libungan, L.A., Steinarsson, A., Marteinsdóttir, G., 2009. Evidence of phenotypic plasticity and local adaption in metabolic rates between components of the Icelandic cod (Gadus morhua L.) stock. Environ. Biol. Fishes 86, 361-370.

Groothuis, T.G.G., Carere, C., 2005. Avian personalities: characterization and epigenesist. Neurosci. Biobehav. Rev. 29, 137-150.

Höglund, E., Sørensen, C., Bakke, M.J., Nilsson, G.E., Øverli, Ø., 2007. Attenuation of stress-induced anorexia in brown trout (Salmo trutta) by pre-treatment with dietary L-tryptophan. Br. J. Nutr. 97, 786789.

Huntingford, F.A., Adams, C.E., 2005. Behavioural syndromes in farmed fish: implications for production and welfare. Behaviour 142, 1207-1221.

Huntingford, F.A., Adams, C., Braithwaite, V.A., Kadri, S., Pottinger, T.G., Sandøe, P., Irwin, S., Kenny, A.P., O'Halloran, J., Fitzgerald, R.D., Duggan, P.F., 1999. Adaptation and validation of a radioimmunoassay kit for measuring plasma cortisol in turbot. Comp. Biochem. Physiol. C 124, 27-31.

Kristiansen, T.S., Ferno, A., 2007. Individual behaviour and growth of halibut (Hippoglossus hippoglossus L.) fed sinking and floating feed: Evidence of different coping styles. Appl. Anim. Behav. Sci. 104, 236-250.

Koolhaas, J.M., Korte, S.M., De Boer, S.F., Van Der Vegt, B.J., Van Reenen, C.G., Hopster, H., De Jong, I.C., Ruis, M.A.W., Blokhuis, H.J., 1999. Coping styles in animals: current status in behavior and stress-physiology. Neurosci. Biobehav. Rev. 23, 925-935.

Lazarus, R.S., 1991. Progress on a cognitive-motivational-relational theory of emotion. Am. Psychol. 46, 819-834.

MacKenzie, S., Ribas, L., Pilarczyk, M., Capdevila, D.M., Kadri, S., Huntingford, F.A., 2009. Screening for coping style increases the power of gene expression studies. PLoS One 4, e5314.

Martins, C.I.M., 2005. Individual variation in growth of African catfish Clarias gariepinus: a search for explanatory factors. PhD thesis. Wageningen University, The Netherlands. $166 \mathrm{pp}$.

Martins, C.I.M., Schrama, J.W., Verreth, J.A.J., 2005. The consistency of individual differences in growth, feed efficiency and feeding behaviour in African catfish Clarias gariepinus (Burchell, 1822) housed individually. Aquacult. Res. 36, 1509-1516.

Martins, C.I.M., Schrama, J.W., Verreth, J.A.J., 2006. The relationship between feed efficiency and stress response in African catfish Clarias gariepinus. Aquaculture 256, 588-595.

Martins, C.I.M., Hillen, B., Schrama, J.W., Verreth, J.A.J., 2008. A brief note on the relationship between residual feed intake and aggression behaviour in juveniles of African catfish Clarias gariepinus. Appl. Anim. Behav. Sci. 111, 408-413.

Minderman, J., Reid, J.M., Evans, P.G.H., Whittingham, M.J., 2009. Personality traits in wild starlings: exploration behavior and environmental sensitivity. Behav. Ecol. 20, 830-837.

Øverli, Ø., Pottinger, T.G., Carrick, T.R., Øverli, E., Winberg, S., 2002. Differences in behaviour between rainbow trout selected for high- and low-stress responsiveness. J. Exp. Biol. 205, 391-395.

Øverli, Ø., Korzan, W.J., Höglund, E., Winberg, S., Bollig, H., Watt, M., Forster, G.L., Barton, B.A., Øverli, E., Renner, K.J., Summers, C.H., 2004. Stress coping style predicts aggression and social dominance in rainbow trout. Horm. Behav. 45, 235-241. 
Øverli, Ø., Winberg, S., Pottinger, T.G., 2005. Behavioral and neuroendocrine correlates of selection for stress responsiveness in rainbow trout-a review. Integr. Comp. Biol. 45, 463-474.

Øverli, Ø., Sørensen, C., Nilsson, G.E., 2006. Behavioral indicators of stresscoping style in rainbow trout: do males and females react differently to novelty? Physiol. Behav. 87, 506-512.

Øverli, Ø., Sørensen, C., Pulman, K.G.T., Pottinger, T.G., Korzan, W., Summers, C.H., Nilsson, G.E., 2007. Evolutionary background for stress-coping styles: relationships between physiological, behavioral, and cognitive traits in non-mammalian vertebrates. Neurosci. Biobehav. Rev. 31, 396-412.

Quinn, J.L., Cresswell, W., 2005. Personality, anti-predation behaviour and behavioural plasticity in the chaffinch Fringilla coelebs. Behaviour 142, 1377-1402.

Ramsay, J.M., Feist, G.W., Varga, Z.M., Westerfield, M., Kent, M.L., Schreck, C.B., 2009. Whole-body cortisol response of zebrafish to acute net handling stress. Aquaculture 297, 157-162.
Reefmann, N., Wechsler, B., Gygax, L., 2009. Behavioural and physiological assessment of positive and negative emotion in sheep. Anim. Behav. 78, 651-659.

Richards, A., French, C.C., Johnson, W., Naparstek, J., Williams, J., 1992. Effects of mood manipulation and anxiety on performance of an emotional Stroop task. Br. J. Psychol. 83 (Pt 4).

Seppänen, E., Kuukka, H., Huuskonen, H., Piironen, J., 2008. Relationship between standard metabolic rate and parasite-induced cataract of juveniles in three Atlantic salmon stocks. J. Fish Biol. 72, 16591674.

Silva, P.I.M., Martins, C.I.M., Engrola, S., Marino, G., Øverli, Ø., Conceição, L.E.C., 2010. Individual differences in cortisol levels and behaviour of Senegalese sole (Solea senegalensis) juveniles: evidence for coping styles. Appl. Anim. Behav. Sci. 124, 75-81.

Verbeek, P., Iwamoto, T., Murakami, N., 2008. Variable stressresponsiveness in wild type and domesticated fighting fish. Physiol. Behav. 93, 83-88. 